\title{
Interne Beratung
}

\author{
Falko von Ameln
}

Online publiziert: 11. Februar 2015

(C) Springer Fachmedien Wiesbaden 2015

Organisationsberatung ist eine von einer externen Instanz erbrachte Dienstleistung - diese Prämisse bestimmt den Diskurs nach wie vor, sei es als explizites Definitionsmerkmal des Beratungsbegriffs, sei es indem interne Beratung durch das Attribut „intern“ als Sonderfall ausgewiesen wird. Die Wirklichkeit sieht jedoch schon lange ganz anders aus: Ein beträchtlicher Teil der früher extern eingekauften Beratungsdienstleistungen wird mittlerweile intern erbracht - die Studie von Galal et al. (2010) nennt einen Wert von $40 \%$, Tendenz weiterhin steigend.

Dennoch ist in der Literatur zur internen Beratung die außenstehende Beobachtungsperspektive nach wie vor prägend - ein Beispiel dafür sind etwa Publikationen zur „Klientenprofessionalisierung“ (Mohe 2003; Mohe und Kolbeck 2003), die einen Kontrapunkt zur Einseitigkeit der überkommenen Perspektive zu setzen beabsichtigen, durch die Wahl der Begrifflichkeit aber gerade die Perspektive fortschreiben, in der Organisationen nur als „Klienten“ einer externen Beratung vorkommen. Spezifische Literatur zum Thema interne Beratung ist dünn gesät: Die mittlerweile zahlreich vorliegenden Praxisberichte (z. B. in Moscho und Richter 2010; Niedereichholz 2000; OrganisationsEntwicklung 2010 oder Personalführung 2007) tragen oft eher den Charakter von erfolgsbeseelten Werbetexten zum Zwecke der Personalrekrutierung oder zur Karriereförderung der Autor/innen. Neben einigen Studien zu strukturellen Merkmalen interner Beratungen (z. B. Galal et al. 2010; Hoyer 2000 oder Leker et al. 2007) sind differenzierte, theoretisch fundierte Auseinandersetzungen mit dem Thema sowie empirische Arbeiten zu beratungswissenschaftlich relevanten Aspekten interner Beratung Mangelware (als positives Beispiel sei allerdings der ausführliche Band von Krizanits 2011 genannt). In Anbetracht dieser Situation gehen Deelmann et al. (2007, S. 230) sogar so weit, von einem „Forschungsversagen“ zu sprechen.

F. von Ameln $(\bowtie)$

Norden, Deutschland

E-Mail: von.ameln@gmx.de 
Der Anspruch des vorliegenden Themenheftes kann natürlich nicht darin liegen, dieses Defizit zu beheben. Vielmehr geht es darum, interne Beratung im Hinblick auf ihre Verortung in den organisationalen Dynamiken zu reflektieren und dabei die mögliche Bandbreite interner Beratung wenn schon nicht vollständig abzubilden, so doch wenigstens über den sonst meist dominanten Bezugsrahmen von Profit-Unternehmen hinaus aufzuzeigen. Die Beiträge verstehen sich als an der Schnittstelle von Theorie und Praxis angesiedelt und richten sich somit an Praktiker/innen und wissenschaftliche Leser/innen gleichermaßen. Die verschiedene Beiträgen nehmen dabei eine jeweils unterschiedliche Gewichtung von Theorie und Praxis vor: Während die in diesem Themenheft versammelten Hauptbeiträge Aufgaben, Chancen und Spannungsfelder interner Beratung von einem eher theoretischen Standpunkt aus beschreiben, beleuchten Interviews mit Leiterinnen und Leitern von Inhouse Consulting-Einheiten mögliche Praxis interner Beratung.

Das Heft wird von einem konzeptuell ausgerichteten Beitrag eröffnet, der mögliche Zukunftsperspektiven für interne Beratung vor dem Hintergrund wachsender Anforderungen an organisationales Lernen aufzeigt. Dabei wird interner Beratung die Funktion zugeschrieben, organisationale Lernprozesse zu koordinieren und dabei sowohl zentralen Steuerungsnotwendigkeiten als auch lokalen Rationalitäten Rechnung zu tragen.

Gundo Lames beschreibt die Entwicklungslinien der Gemeindeberatung als interner Beratung der Kirchen. Dabei geht er besonders auf ein typisches Konfliktfeld interner Beratung ein, nämlich mögliche Loyalitätskonflikte gegenüber der lokalen Organisationseinheit (in diesem Fall der Gemeinde vor Ort) auf der einen Seite und der Kirchenleitung als Auftraggeber auf der anderen Seite. Sein Beitrag zeigt auch, wie sich pastorale und organisationsentwicklerische Diskurse in der Gemeindeberatung in den vergangenen Jahrzehnten (nicht immer konfliktfrei) ergänzt und verschränkt haben.

Auch im nachfolgenden Interview mit Andreas Einig, der den Aufbau einer Inhouse-Consulting in einem kirchlichen Unternehmen des Gesundheits- und Sozialwesens beschreibt, wird diese Fragestellung aufgegriffen. Einig beschreibt die Funktion von Inhouse Consulting mit Bezug auf Metz' Definition von Religion als Herstellung von Unterbrechungen und zeigt Chancen für das Inhouse Consulting in einer Kultur auf, in der die Unterbrechung des Tagesgeschäfts zum Zwecke der Reflexion durch die Grundwerte des Unternehmens begünstigt wird. Das Interview zeigt auch, wie Inhouse Consulting in einer Expertenorganisation dazu beitragen kann, den Dialog zwischen den verschiedenen Berufsgruppen zu fördern.

Tamara Kuhn und C. Benjamin Nakhosteen geben mit ihrem Artikel einen Einblick in die Arbeit des Inhouse Consulting-Teams bei der ThyssenKrupp Steel Europe AG. Sie argumentieren, dass soziale Identität zu den Schlüsseldimensionen der lernenden Organisation gehört und begründen die besonderen Chancen der internen Beratung vor dem Hintergrund der Theorie der sozialen Identität.

Simone Steinberg beschreibt im Interview die vielfältige Praxis einer internen Beratungseinheit mit psychologischem Schwerpunkt bei der Deutschen Bahn, deren Angebotsportfolio von Change-Begleitung über Trainingsangebote für Fach- und Führungskräfte und Managementdiagnostik bis hin zu Gesundheitsmanagement und Traumaprävention reicht. Die im dieses Heft eröffnenden Grundlagenbeitrag ange- 
sprochene Frage, wie sich Veränderungskompetenz in komplexen Organisationen und Organisationsumwelten verstetigen lässt, klingt in diesem Interview wieder an.

Der Beitrag von Astrid Schreyögg versteht sich als umfangreiche Einführung in das organisationsinterne Coaching. Ihr Text stellt Typen des internen Coachings vor, beschreibt Ziele und Funktionen internen Coachings, behandelt eine Reihe von praxisrelevanten Aspekten (z. B. Regelungen bestehen für die Wahl von Coaches, Implementierungsstrategien, Qualitätssicherung) und thematisiert typische Fallstricke internen Coachings.

Der abschließende Beitrag von Beatrix und Johannes Wildt skizziert, wie sich eine hochschuldidaktisch moderierte Curriculumentwicklung als eine Form der Organisationsentwicklung an Hochschulen herausgebildet hat. Auf der Basis einer ausführlichen Analyse der Funktionen und Chancen entwickeln die Autor/innen eine Zukunftsvision einer internen Beratung an Hochschulen.

Zwei weitere geplante Interviews fanden leider nicht ihren Weg in dieses Heft: Ein bereits geführtes und transkribiertes Interview mit der internen Beraterin eines Großunternehmens wurde zurückgezogen, ein weiteres Interview mit der Leitung der internen Beratungseinheit eines Versicherungskonzerns fand wegen eines Zuständigkeitswechsels nicht statt.

Der Herausgeber und die Redaktion der Zeitschrift wünschen allen Leser/innen eine anregende und erkenntnisreiche Lektüre.

Falko von Ameln

\section{Literatur}

Deelmann, T. A., Huchler, A., Jansen, S. A., \& Petmecky, A. (2007). Interne Unternehmensberatungen. Thesen und erste Tests für den Entwurf einer Theorie der Internen Beratung. Zeitschrift für Management, 3(2), 228-266.

Galal, K., Richter, A., \& Steinbock, K. (2010). Inhouse-Beratung in Deutschland: Ergebnisse einer empirischen Studie. In A. Moscho \& A. Richter (Hrsg.), Inhouse-Consulting in Deutschland. Markt, Strukturen, Strategien (S. 11-30). Wiesbaden: Gabler.

Hoyer, H. (2000). Internes Consulting in Deutschland - Ergebnisse einer Marktuntersuchung. In C. Niedereichholz (Hrsg.), Internes Consulting: Grundlagen-Praxisbeispiele-Spezialthemen (S. 55-81). München: Oldenbourg.

Krizanits, J. (2011). Professionsfeld Inhouse Consulting. Praxis und Theorie der internen Organisationsberatung. Heidelberg: Carl-Auer.

Mohe, M. (2003). Klientenprofessionalisierung. Marburg: Metropolis.

Mohe, M., \& Kolbeck, C. (2003). Klientenprofessionalisierung in Deutschland. Stand des professionellen Umgangs mit Beratung bei deutschen Dax-und MDax-Unternehmen. Empirische Ergebnisse, Best Practices und strategische Implikationen. Oldenburg: Carl-von-Ossietzky-Universität.

Moscho, A., \& Richter, A. (Hrsg.). (2010). Inhouse-Consulting in Deutschland. Markt, Strukturen, Strategien. Wiesbaden: Gabler.

Niedereichholz, C. (Hrsg.). (2000). Internes Consulting: Grundlagen-Praxisbeispiele - Spezialthemen. München: Oldenbourg.

OrganisationsEntwicklung. (2010). Themenheft „Champions oder Loser? Balanceakt interne Beratung“. 29(2).

Personalführung. (2007). Themenschwerpunkt Inhouse Consulting. Heft 40(5). 